\title{
Rotational velocities of single and binary O-type stars in the Tarantula Nebula
}

\section{O. H. Ramírez-Agudelo ${ }^{1}$, H. Sana ${ }^{2}$, A. de Koter $^{1,3}$, S. Simón-Díaz ${ }^{4,5}$, S. E. de Mink ${ }^{6,7}$, F. Tramper ${ }^{1}$, P. L. Dufton ${ }^{8}$, C. J. Evans ${ }^{9}$, G. Gräfener ${ }^{10}$, A. Herrero ${ }^{4,5}$, N. Langer ${ }^{11}$, D. J. Lennon ${ }^{12}$, J. Maíz Apellániz ${ }^{13}$, N. Markova ${ }^{14}$, F. Najarro ${ }^{15}$, J. Puls ${ }^{16}$, W.D. Taylor ${ }^{9}$ and J.S. Vink ${ }^{10}$,}

${ }^{1}$ Astronomical Institute Anton Pannekoek, University of Amsterdam, The Netherlands email: o.h.ramirezagudelo@uva.nl

${ }^{2}$ ESA/Space Telescope Science Institute 3700 San Martin Drive, Baltimore, MD21218, USA

${ }^{3}$ Instituut voor Sterrenkunde, Universiteit Leuven, Celestijnenlaan 200D, 3001, Leuven, Belgium

${ }^{4}$ Instituto de Astrofísica de Canarias, C/ Vía Láctea s/n, E-38200 La Laguna, Tenerife, Spain

${ }^{5}$ Departamento de Astrofísica, Universidad de La Laguna, Avda. Astrofísico Francisco Sánchez s/n, E-38071 La Laguna, Tenerife, Spain

${ }^{6}$ Observatories of the Carnegie Institution for Science, 813 Santa Barbara St, Pasadena, CA 91101, USA

${ }^{7}$ Cahill Center for Astrophysics, California Institute of Technology, Pasadena, CA 91125, USA

${ }^{8}$ Astrophysics Research Centre, School of Mathematics and Physics, Queen's University of Belfast, Belfast BT7 1NN, UK

${ }^{9}$ UK Astronomy Technology Centre, Royal Observatory Edinburgh, Blackford Hill, Edinburgh, EH9 3HJ, UK

${ }^{10}$ Armagh Observatory, College Hill, Armagh, BT61 9DG, Northern Ireland, UK

${ }^{11}$ Argelander-Institut für Astronomie, Universität Bonn, Auf dem Hügel 71, 53121 Bonn, Germany

${ }^{12}$ European Space Agency, European Space Astronomy Centre, Camino Bajo del Castillo s/n, Urbanizacin Villafranca del Castillo, 28691 Villanueva de la Caada, Madrid, Spain

${ }^{13}$ Instituto de Astrofísica de Andalucía-CSIC, Glorieta de la Astronomía s/n, E-18008 Granada, Spain

${ }^{14}$ Institute of Astronomy with NAO, Bulgarian Academy of Science, PO Box 136, 4700 Smoljan, Bulgaria

${ }^{15}$ Centro de Astrobiología (CSIC-INTA), Ctra. de Torrejón a Ajalvir km-4, E-28850 Torrejón de Ardoz, Madrid, Spain

${ }^{16}$ Universitätssternwarte, Scheinerstrasse 1, 81679 München, Germany

\begin{abstract}
Rotation is a key parameter in the evolution of massive stars, affecting their evolution, chemical yields, ionizing photon budget, and final fate. We determined the projected rotational velocity, $v_{e} \sin i$, of $\sim 330$ O-type objects, i.e. $\sim 210$ spectroscopic single stars and $\sim 110$ primaries in binary systems, in the Tarantula nebula or 30 Doradus (30 Dor) region. The observations were taken using VLT/FLAMES and constitute the largest homogeneous dataset of multi-epoch spectroscopy of O-type stars currently available. The most distinctive feature of the $v_{e} \sin i$ distributions of the presumed-single stars and primaries in 30 Dor is a low-velocity peak at around $100 \mathrm{~km} \mathrm{~s}^{-1}$. Stellar winds are not expected to have spun-down the bulk of the stars significantly since their arrival on the main sequence and therefore the peak in the single star sample is likely to represent the outcome of the formation process. Whereas the spin distribution of presumed-single stars shows a well developed tail of stars rotating more rapidly than $300 \mathrm{~km} \mathrm{~s}^{-1}$, the sample of primaries does not feature such a high-velocity tail. The tail of the
\end{abstract}


presumed-single star distribution is attributed for the most part - and could potentially be completely due - to spun-up binary products that appear as single stars or that have merged. This would be consistent with the lack of such post-interaction products in the binary sample, that is expected to be dominated by pre-interaction systems. The peak in this distribution is broader and is shifted toward somewhat higher spin rates compared to the distribution of presumedsingle stars. Systems displaying large radial velocity variations, typical for short period systems, appear mostly responsible for these differences.

Keywords. stars: rotation, stars: binaries, galaxies: Magellanic Clouds

\section{Introduction}

Rotation impacts the evolution of massive stars, affecting their evolution, chemical yields, budget of ionizing photons and final fate as supernovae and long gamma-ray bursts (e.g. Langer 2012). For massive stars the initial distribution of spin rates is especially interesting because so little is known about how these stars form (e.g., Zinnecker \& Yorke 2007). Potentially, it can tell us more about the formation process.

The 30 Dor starburst region in the Large Magellanic Cloud contains the richest sample of massive stars in the Local Group and is the best possible laboratory to investigate aspects of the formation and evolution of massive stars, and to establish statistically meaningful distributions of their physical properties. Here we present an analysis of the $v_{e} \sin i$ properties of the O-type objects, i.e. spectroscopic single stars and binary systems observed in the context of the VLT-FLAMES Tarantula Survey (VFTS; Evans et al. 2011; de Koter et al. 2011). VFTS is a multi-epoch spectroscopic campaign targeting over 300 O-type objects - singles and binaries. The spectral classification and radial velocity (RV) measurements, relevant for the study at hand, are presented in Walborn et al. (2014) and Sana et al. (2013). Here we will report on the projected rotational properties, $v_{e} \sin i$, of the presumed-single O-type stars (Ramírez-Agudelo et al. 2013; RamirezAgudelo \& VFTS Consortium 2013) and primary stars, i.e. the brightest component of binary systems composed of at least one O-type star (Ramírez-Agudelo et al. in prep.).

\section{Sample and Method}

The VFTS project and the data have been described in Evans et al. (2011). In short the total Medusa sample contains 330 O-type objects. Sana et al. (2013), from multiepoch radial velocity $(\mathrm{RV})$ measurements have identified $\sim 210$ O-type stars that show no significant $R V$ variations $(\Delta R V)$ and are presumably single $\left(\Delta R V \leqslant 20 \mathrm{~km} \mathrm{~s}^{-1}\right)$ and $\sim 110$ objects (the rest of the sample) with $\Delta \mathrm{RV}>20 \mathrm{~km} \mathrm{~s}^{-1}$ that are considered spectroscopic binaries.

For the single sample we use Fourier transform (Gray 1976; Simón-Díaz \& Herrero 2007) and line profile fitting methods (Simón-Díaz \& Herrero 2014) to measure projected rotational velocities. A discussion of the methods used and the accuracy that can be achieved can be found in Ramírez-Agudelo et al. (2013). To obtain the projected rotational velocities of the binary sample we calibrate full width at half maximum (FWHM) measurements versus $v_{e} \sin i$ for specific spectral lines, using the FWHM measurements of the O-type stars from Sana et al. (2013) and the $v_{e} \sin i$ measurements of the single O-type star presented in Ramírez-Agudelo et al. (2013). Details of the method and its accuracy are found in Ramírez-Agudelo et al. in prep.. 


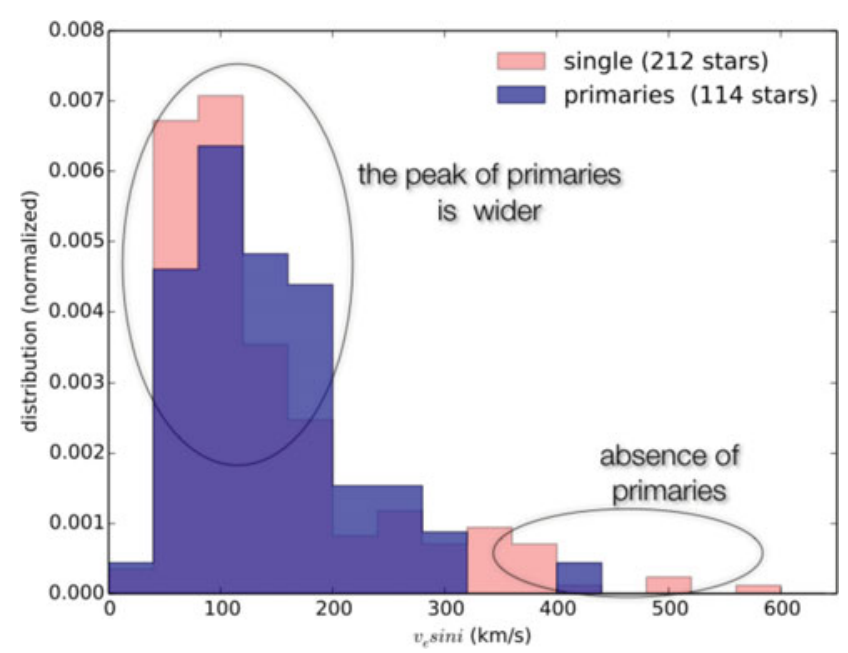

Figure 1. Distribution of the projected rotational velocities, $v_{e} \sin i$, of the O-type presumably single and primary samples.

\section{Results}

Figure 1 shows the $v_{e} \sin i$ distributions of presumed-single stars and primaries. Qualitatively, both distributions display a peak at around $\sim 100 \mathrm{~km} \mathrm{~s}^{-1}$ though we also note some differences. First, the main peak of the primary distribution is wider than that of the presumed-single sample. Second, at $v_{e} \sin i>300 \mathrm{~km} \mathrm{~s}^{-1}$ there is a deficiency of rapidly rotating primaries with respect to the presumed-single stars. While for the presumed-single sample 22 stars out of 212 exhibit projected rotational velocities larger than $300 \mathrm{~km} \mathrm{~s}^{-1}$ (corresponding to $10 \pm 2 \%$ of that sample), in the primaries we only

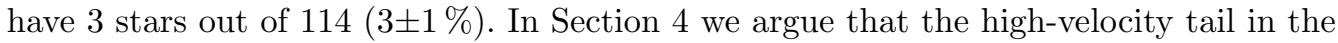
presumed-single star distribution is compatible with post-interaction binary evolution.

The size of the primary sample allows us to explore $v_{e} \sin i$ distributions for subpopulations. By selecting a radial velocity amplitude limit $\left(\Delta \mathrm{RV}_{\text {limit }}\right)$ of $200 \mathrm{~km} \mathrm{~s}^{-1}$ we divide the primary sample into two subsamples, i.e. stars that display $\Delta R V \leqslant \Delta R V_{\text {limit }}$ (henceforth low- $\Delta R V$ ) and stars with $\Delta R V>\Delta R V_{\text {limit }}$ (high- $\Delta R V$ ). The latter are systems where we expect tidal synchronization to become important during the main sequence phase; given our sample properties they roughly correspond to binaries with an orbital period of less than 10 days (see Ramírez-Agudelo et al. in prep.). Most of the systems in the low- $\Delta R V$ subsample are wider systems that will not suffer from tides with the potential exception of those that are seen at a low inclination angle. Figure 2 plots the $v_{e} \sin i$ distributions of the low- $\Delta \mathrm{RV}$ (85 stars) and high- $\Delta \mathrm{RV}$ (29 stars) subsamples. At $v_{e} \sin i \leqslant 200 \mathrm{~km} \mathrm{~s}^{-1}$ the distribution of high- $\Delta \mathrm{RV}$ sources, compared to the low- $\Delta \mathrm{RV}$ sources, appears shifted by one bin to higher rotational velocities. The weighted mean of the samples (119 and $190 \mathrm{~km} \mathrm{~s}^{-1}$ for the low- and high- $\Delta \mathrm{RV}$ samples respectively) are significantly different from one another, confirming an average faster rotation for the high- $\Delta$ RV (short periods) systems. These differences may be related to tidal effects, an hypothesis that we will explore further in the next subsection.

\subsection{Tidal interaction}

To investigate the effect of tidal locking in our sample we make use of a diagram showing the relation between projected rotational velocity and amplitude of the RV variations 


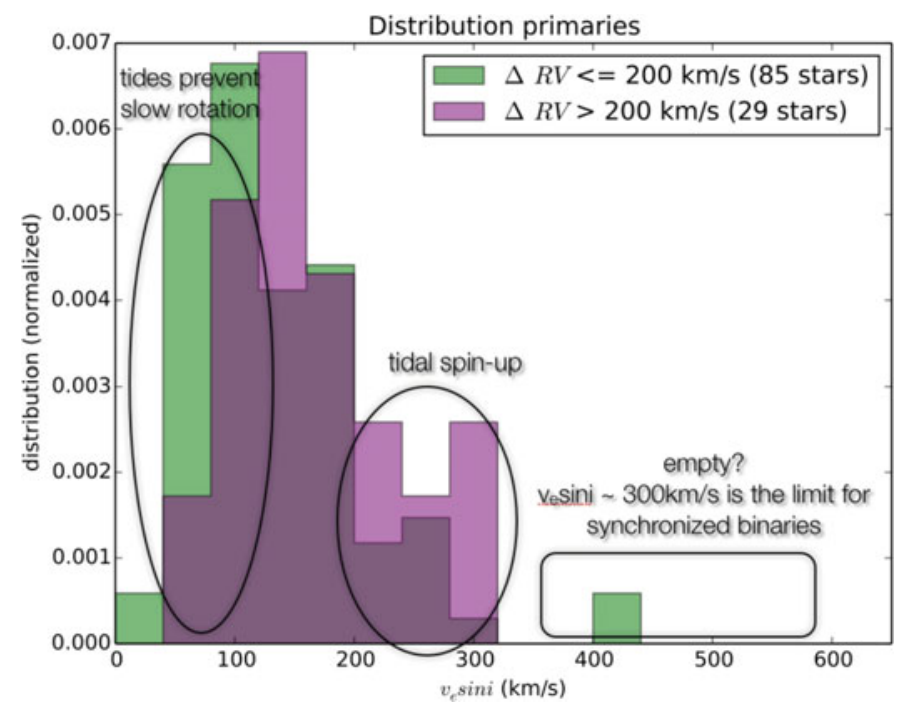

Figure 2. Distribution of the projected rotational velocities, $v_{e} \sin i$, of the O-type primaries with $\Delta \mathrm{RV} \leqslant \Delta \mathrm{RV}_{\text {limit }}($ low- $\Delta \mathrm{RV})$ and $\Delta \mathrm{RV}>\Delta \mathrm{RV}_{\text {limit }}$ (high- $\left.\Delta \mathrm{RV}\right)$.

(see Fig. 3). The timescale of tidal synchronization is a function of the primary mass $M_{1}$, the mass ratio of the primary and secondary $q=M_{2} / M_{1}$, and the orbital period $P_{\text {orb }}$. For our sample we lack information about the latter and therefore we use the radial velocity amplitude $\Delta \mathrm{RV}$ as a proxy of the period. Figure 3 gives the relation between $v_{e} \sin i$ and $\Delta \mathrm{RV}$ for the sample of primaries. The green dashed region shows the range of parameter space for which tidal synchronization of a $20 M_{\odot}$ primary - a typical mass for our sample - occurs within the main sequence life for $q$ ranging from 0.25 (left-side boundary) to 1 (right-side boundary) and $P_{\text {orb }}$ ranging from $10 \mathrm{~d}$ (bottom boundary) to $0.25 \mathrm{~d}$ (upper boundary), or sooner when the primary fills its Roche lobe prior to reaching the end of the main sequence (de Mink et al. 2009, 2013). The thick green line is for $q=0.5$. The top axis of the figure displays the orbital period $P_{\text {orb }} / \sin i$ associated with the radial velocity amplitude given on the horizontal axis, assuming $\Delta R V$ is twice the actual semi-amplitude of the radial velocity curve. Similarly, the $P_{\mathrm{rot}} / \sin i$ displayed on the right vertical axis corresponds to the projected rotational velocity given on the left.

Tides are less effective for smaller mass ratios and longer periods, therefore the timescale of tidal synchronization is longest for systems in the lower left corner of the green zone. In the upper right corner synchronization proceeds the fastest. For instance, for a $0.5 \mathrm{~d}$ system with $q=0.75$ synchronization by turbulent viscosity and radiative dissipation occurs within about 1 percent of the main sequence lifetime (de Mink et al. 2009). The gray region shows the $M_{1}$ dependence by also displaying the zone as defined here for a $60 M_{\odot}$ primary. It is stretched out to both higher $v_{e} \sin i(\propto R)$, and $\Delta R V\left(\propto M_{1}^{1 / 3}\right)$, where $R$ is the stellar radius. The green zone that according to the above argument may be expected to contain systems that are synchronized or tend toward synchronization is populated by 40 primaries. The 29 sources that constitute the pink $(\Delta R V>$ $200 \mathrm{~km} \mathrm{~s}^{-1}$ ) distribution in Fig. 2 are almost exclusively found in the green region, more specifically in the part of that region for which synchronization by tides is the most relevant. 


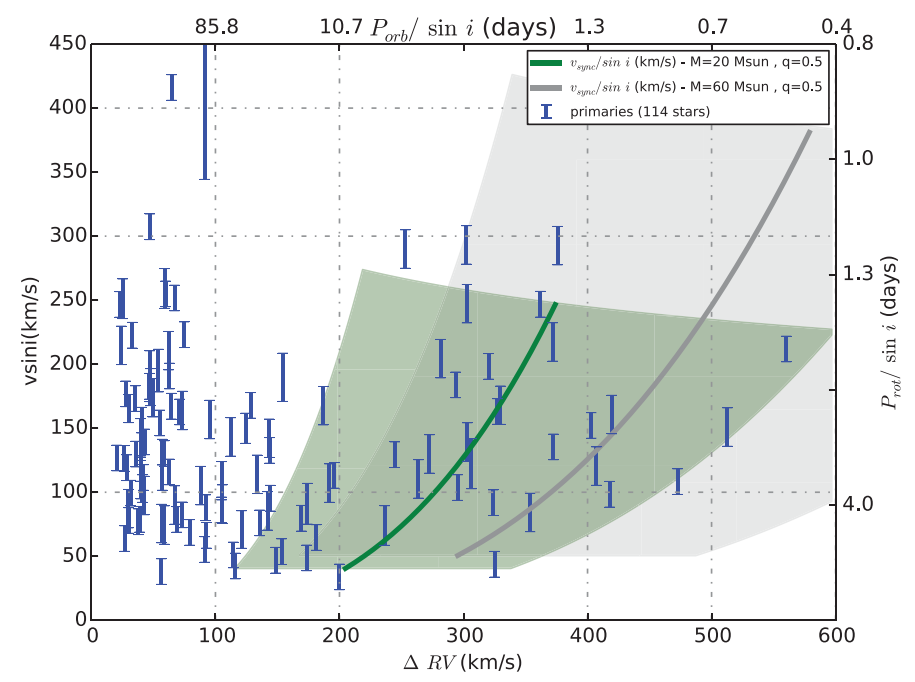

Figure 3. Projected rotational velocity vs. radial velocity amplitude for the primary sample (114 stars). The green and gray regions show where a $20 M_{\odot}$ and $60 M_{\odot}$ primary is expected to become synchronized with its companion before the primary leaves the main sequence, computed for mass ratios $q$ ranging between 0.25 and 1 and periods $P_{\text {orb }}$ from 0.25 to 10 days. The thick green and gray lines are for $q=0.5$. The top axis displays the orbital period $P_{\text {orb }} / \sin i$ associated with the radial velocity amplitude given on the horizontal axis for a $20 M_{\odot}$ primary. Similarly, $P_{\text {rot }} / \sin i$ displayed on the right vertical axis corresponds to the projected rotational velocity given on the left.

\section{Implications}

The most distinctive feature of the $v_{e} \sin i$ distribution of the O-type presumed-single stars and primaries in 30 Dor is its low-velocity peak at around $100 \mathrm{~km} \mathrm{~s}^{-1}$. For the bulk of the samples, mass loss in a stellar wind and/or envelope expansion is not efficient enough to significantly spin down these stars. Therefore the peak is likely to be the outcome of the formation process (see discussion in Ramírez-Agudelo et al. 2013 and in prep.). The presence of a high-velocity tail in the presumed-single sample, and the absence of such a tail in the primary sample, is compatible with predictions of binary interaction. Rapid rotators result from spin-up through mass transfer and mergers (de Mink et al. 2013), that mostly appear as, or have become, single objects (de Mink et al. 2014). Such a nature of the high-velocity tail has important implications for the evolutionary origin of the progenitors of long gamma-ray bursts, reducing the likelihood that long-GRBs may also result from single stars that are born spinning rapidly. Finally, if post-interaction systems have been removed from the binary sample for the above outlined reason it is dominated by pre-interaction systems. The short period systems among these may suffer from tidal synchronization effects that may qualitatively explain the differences in the low-velocity peak structure of presumed-single stars and of primaries.

\section{References}

de Koter, A., Sana, H., Evans, C. J., et al. 2011, Journal of Physics Conference Series 328(1), 012022

de Mink, S. E., Cantiello, M., Langer, N., et al. 2009, A\& A 497, 243

de Mink, S. E., Langer, N., Izzard, R. G., Sana, H., \& de Koter, A. 2013, ApJ 764, 166

de Mink, S. E., Sana, H., Langer, N., Izzard, R. G., \& Schneider, F. R. N. 2014, ApJ 782, 7

Evans, C. J., Taylor, W. D., Hénault-Brunet, V., et al. 2011, A 6 A 530, A108 
Gray, D. 1976, The Observation and Analysis of Stellar Photospheres, Cambridge University Press, third edition edition

Langer, N. 2012, ARA\&A 50, 107

Ramírez-Agudelo, O. H., Simón-Díaz, S., Sana, H., et al. 2013, A\&3A 560, A29

Ramirez-Agudelo, O. H. \& VFTS Consortium 2013, in Massive Stars: From alpha to Omega

Sana, H., de Koter, A., de Mink, S. E., et al. 2013, A\&A 550, A107

Simón-Díaz, S. \& Herrero, A. 2007, A\&\&A 468, 1063

Simón-Díaz, S. \& Herrero, A. 2014, A\& A 562, A135

Townsend, R. H. D., Owocki, S. P., \& Howarth, I. D. 2004, MNRAS 350, 189

Walborn, N. R., Sana, H., Simón-Díaz, S., et al. 2014, A\&A 564, A40

Zinnecker, H. \& Yorke, H. W. 2007, ARA\& A 45, 481

\section{Discussion}

MAssey: Conti \& Ebbets (1977) showed that the dwarf O stars had both a low and a high $v_{e} \sin i$ peak but that the $\mathrm{O}$ supergiants have only a low peak. How can post interacting $\mathrm{O}$ stars (merged or not) be luminosity class $\mathrm{V}$ with modest $\mathrm{M}_{\mathrm{v}}$ ? Would not you expect them to have high luminosities?

RAmíRez-Agudelo: In Ramírez-Agudelo et al. (2013) subdivide the contribution of dwarfs, giants and supergiants to the $v_{e} \sin i$ distribution. We find that the tail is dominated by the dwarfs and that supergiants are almost missing. The reason is that the break-up velocity of supergiants is considerably lower, about $300 \mathrm{~km} \mathrm{~s}^{-1}$, than that of dwarfs $\left(\sim 600 \mathrm{~km} \mathrm{~s}^{-1}\right)$, simply because they are bigger. The secondary OV stars that receive the mass may remain dwarfs, the interaction itself does not turn them into supergiants. Later they may evolve towards supergiants - but then their $v_{e} \sin i$ will decrease because of envelope expansion.

MAEDER: You have shown that you are calibrating your $v_{e} \sin i$ on the basis of the change of the line profiles. However, as shown long ago by Collins, the equivalent width of the lines may also change since the stellar flux is coming from regions with a variety of $g_{\text {eff }}$ and $\mathrm{T}_{\text {eff }}$. If this is not accounted for, a large underestimate of the $v_{e} \sin i$ may result. Could you please comment on what you are doing.

RAmíREZ-Agudelo: The gravity darkening effect that you refer to has been studied for B stars by Townsend et al. (2004) and becomes important for stars that spin faster than about $95 \%$ of critical. In our sample of presumably-single $\mathrm{O}$ stars only six sources out of the $\sim 210$ spin above $60 \%$ of critical, and one source out of 114 binaries. Because of the very limited effect on the sample as a whole we did not correct for the darkening effect. 